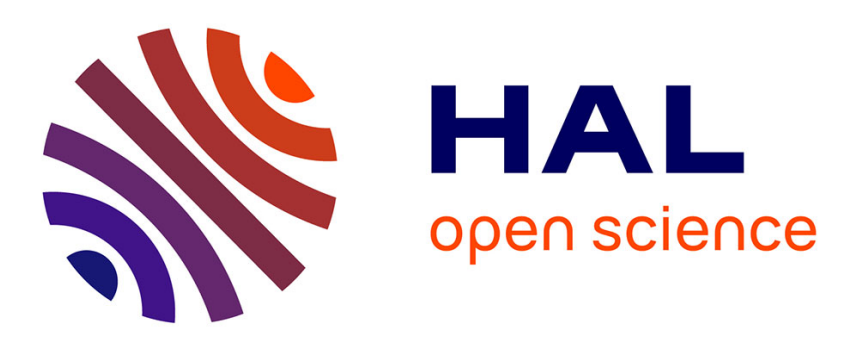

\title{
Réformer la société. Les hommes de la société générale des prisons, 1877-1900 \\ Martine Kaluszynski
}

\section{To cite this version:}

Martine Kaluszynski. Réformer la société. Les hommes de la société générale des prisons, 1877-1900. Genèses. Sciences sociales et histoire, 1997, Etatisations, 28 (28), pp 76-94. 10.3406/genes.1997.1463 . hal-01223174

\section{HAL Id: hal-01223174 \\ https://hal.science/hal-01223174}

Submitted on 2 Nov 2015

HAL is a multi-disciplinary open access archive for the deposit and dissemination of scientific research documents, whether they are published or not. The documents may come from teaching and research institutions in France or abroad, or from public or private research centers.
L'archive ouverte pluridisciplinaire HAL, est destinée au dépôt et à la diffusion de documents scientifiques de niveau recherche, publiés ou non, émanant des établissements d'enseignement et de recherche français ou étrangers, des laboratoires publics ou privés. 


\title{
Réformer la société. Les hommes de la société générale des
} prisons, 1877-1900

In: Genèses, 28, 1997. Étatisations. pp. 76-94.

\begin{abstract}
Résumé
- Martine Kaluszynski: Réformer la société. Les hommes de la Société générale des prisons, 1877-1900 Atelier du politique dans son activité d'expertise et d'élaboration législative, la Société générale des prisons, association d'initiative gouvernementale, a été analysée de 1877 à 1900 à travers les hommes qui la composent. Cette recension permet de caractériser cet espace et d'esquisser «l'esprit» SGP. À travers les parcours et itinéraires de nombreux sociétaires, il est possible de repérer des espaces et des lieux qui ébauchent une cartographie et contribuent à l'idée d'une communauté réformatrice en France à la fin du XIXe siècle. Enfin, par les «professions de foi» de ces hommes se dessine un mode d'exercice du politique fondé sur l'engagement associatif, apolitique, mais qui a néanmoins capacité à bouleverser Tordre social et politique.
\end{abstract}

\section{Abstract}

Martine Kaluzynski: Reforming Society: the members of the General Society of Prisons, 1877-1900 The General Society of Prisons, an association set up on government initiative, was a political workshop in its consulting and legislative preparation activity. In this article, it is analysed from 1877 to 1900 through the biographies of its members. This membership census provides the basis for a description of the sphere and a glimpse of the GSP «spirit». By tracing the careers and itineraries of a number of society members, it is possible to come up with a mapping of the association from the spheres and places involved, and contribute to the idea of a reform community in France at the end of the 19th century. Finally, the "professions of faith» of these men offer a glimpse of a mode of political practice based on apolitical associative commitment, which nevertheless is capable of disrupting the established social and political order.

Citer ce document / Cite this document :

Kaluszynski Martine. Réformer la société. Les hommes de la société générale des prisons, 1877-1900. In: Genèses, $28,1997$. Étatisations. pp. 76-94.

doi : $10.3406 /$ genes.1997.1463

http://www.persee.fr/web/revues/home/prescript/article/genes_1155-3219_1997_num_28_1_1463 
D O S S I E R

Genèses 28, septembre 1997 , pp. $75-93$

\section{RÉFORMER}

\section{LA SOCIÉTÉ}

LES HOMMES

DE LA SOCIÉTÉ GÉNÉRALE

DES PRISONS 1877-1900

\section{Martine Kaluszynski}

1. Voir Jacques-Guy Petit, Ces peines obscures. La prison pénale en France 1780-1875, Paris, Fayard, 1990;

Robert Badinter, La Prison républicaine 1871-1914, Paris, Fayard, 1992.

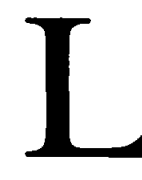

a Société générale des prisons est née en 1877 , deux ans après la loi sur l'emprisonnement cellulaire, elle-même fruit de réflexions issues de l'enquête parlementaire de $1872^{1}$. Cette Société, qui existe encore de nos jours, a connu différents moments dans son évolution. La période républicaine, de 1877 à 1914 , est un moment fort de son histoire si l'on s'attache au rôle que la SGP a joué alors comme laboratoire de la législation pénale. Impulsant, animant les débats, elle est à observer comme un laboratoire d'idées permettant une réflexion approfondie hors du cadre de l'État, en vue d'aboutir à des propositions susceptibles de faire avancer la réforme pénitentiaire grâce à des changements législatifs. La SGP va s'imposer comme une commission extra-parlementaire privée siégeant en permanence. Cette "association d'initiative gouvernementale» dont les statuts sont approuvés par arrêté le 22 mai 1877 et qui est reconnue d'utilité publique en 1889 , va être à la fois un lieu de conception de normes juridiques à travers l'élaboration ou l'utilisation des savoirs sur le crime et un groupe de pression politique menant une action officielle et officieuse.

En instituant des réunions périodiques où sont examinées toutes les questions ayant trait au régime pénitentiaire ou pénal, en assurant la publicité la plus large à ses travaux au moyen d'un bulletin, en apportant son "concours" aux institutions, la Société générale des prisons se veut et va devenir un vaste centre d'études, d'actions et d'informations sur la question pénitentiaire, 
les pénalités et, plus largement, permet d'entrevoir quels sont les problèmes posés aux hommes de ce temps à travers le pénal. Ceci afin d'aboutir à la réalisation de son projet, extrêmement vaste et flou: contribuer à l'amélioration du régime pénitentiaire en France.

Il s'agira ici d'assouvir une réelle curiosité sur cet espace associatif qui se constitue, au-delà des doctrines et des partis, pour réfléchir et agir sur l'élaboration des politiques pénales. Curiosité et interrogation, donc, sur un des lieux de réflexion et d'action dans le champ politique dont une des caractéristiques est de ne pas être «estampillé »: la SGP n'est ni totalement publique ni totalement privée. Le rôle qu'elle tient dans la société républicaine, ses rapports ou résonances avec le milieu de la réforme sociale sont les axes sur lesquels nous allons maintenant tenter de réfléchir.

Dans la filiation de la Société royale des prisons fondée par Louis XVIII ${ }^{2}$, cette Société se veut indépendante de l'administration pénitentiaire comme du Conseil supérieur des prisons et vise à la bonne application de la loi de 1875 , tentant de faire entrer dans les mœurs la réforme pénitentiaire, d'en faciliter et d'en généraliser l'exécution. Elle s'appuie pour cela sur des hommes «éclairés et généreux qui viennent du Parlement, du Commerce, de l'Industrie, de la Magistrature et du Barreau ${ }^{3}$ », pour qui l'association semble être le moyen nécessaire pour faire aboutir un projet de réforme. C'est ce qu'exprimait Léon Lefébure:

"Le succès d'une réforme ne dépend pas du bon vouloir de l'administration. Il est nécessaire que celle-ci trouve dans l'opinion publique un concours moral.»

Et, plus loin, il ajoutait:

"Le moment n'est-il pas venu pour atteindre le but d'une si haute importance sociale de recourir à nouveau à cette force de l'association que nous laissons trop souvent sommeiller dans notre pays? ${ }^{4}$

C'est de ces hommes, de leur présence, de leur statut, que cette recherche est partie, afin de tenter de comprendre l'ossature de cet espace, l'essence de ce projet de réforme pénitentiaire que les élites au XIX $\mathrm{X}^{\mathrm{e}}$ siècle considèrent comme l'un des principaux moyens qui permettra de résoudre la question sociale. Cette «filiation» du pénal au social apparaîtra dans un savoir-faire et des modalités d'organisation ${ }^{5}$ autant que dans la similitude de préoccupations et d'objectifs que portent des hommes qui se trouvent être en quelque sorte «bicéphales ${ }^{6}$.
2. Voir Catherine Duprat, «Punir et guérir. En 1819, la prison des philanthropes", in M. Perrot (éd.). L'Impossible Prison, Paris, 1986, pp. 64-122.

3. Charles Lucas, discours de séance, Revue pénitentiaire (RP), 1877, p. 73.

4. Voir Léon Lefébure, «La Société générale des prisons », $R P ., 1877$, pp. 3-5.

5. Voir Didier Renard, «Le milieu des congrès d'assistance, 1889-1906»; il indique le rôle des congrès pénitentiaires comme «modèle » à l'élaboration d'une structuration dans le domaine de l'Assistance, in Christian Topalov (éd.) Laboratoires du nouveau siècle. La nébuleuse réformatrice et ses réseaux en France, 1880-1920, Paris, EHESS, à paraître.

6. Voir par exemple les figures de L. Lefébure, Emile Cheysson, O. d'Haussonville. 


\section{O S S I E R}

\section{Etatisations}

Martine Kaluszynski Réformer la société

7. Les articles sont très nombreux autour de l'enfance, (thème le plus largement traité) du patronage, du droit de grâce, de la peine de mort, de la détention provisoire, du vagabondage, de la récidive.

\section{Expression d'E. Chcysson, Discours} séance, $R P, 1896$, p. 496.

9. Les sources primaires de ce travail se composent de la liste des membres et des responsables, avec leur statut, et leur adresse, présentée chaque année dans le numéro relié de la Revue pénitentiaire. Matériau fiable, cette liste dressée par les soins du trésorier est imprimée au début de l'année après avoir été soumise au conseil de direction qui en arrête la forme définitive. Douze séries homogènes ont été traitées intégralement et les autres séries traitées partiellement. 1493 notices ont été établies pour cette période, dont 742 pour Paris, 338 pour la province, 58 pour Paris et la province, 365 pour l'étranger.

10. La coupure de 1900 n'est pas la plus pertinente au vu de l'évolution observée au sein de la société. Cette borne chronologique symbolique est due à l'état d'avancement actuel du travail de recension, mais elle offre néanmoins vingt-trois années d'observation et permet de mettre en évidence des «tendances lourdes» que la poursuite du travail permettra d'affiner et de nuancer.

11. Voir Philippe Levillain, «Les protagonistes: de la biographie», in R. Rémond (éd.), Pour une histoire politique. Paris, Éd. du Seuil, 1988, pp. 121 159.

12. L'État est pénétré par la société de la même façon qu'il entretient des liens étroits avec elle. Comme le suggère François-Xavier Merrien: «L'État n'est pas un pur appareil de régulation de la société [...] L'attention doit se porter sur ces réseaux plus ou moins structurés et hiérarchisés des relations entre l'État et la société civile: réseaux par lesquels des groupes ayant accès au pouvoir d'État cherchent à consolider leur influence ou leurs privilèges personnels et/ou réseaux par lesquels des membres de l'État cherchent à influencer les segments stratégiques de la société civile à des fins publiques ou personnelles. »,
L'analyse thématique de la Revue pénitentiaire, organe de la Société, montre que la place accordée à la prison, objet originel de réflexions, régresse au profit des questions pénales, de discussions plus générales sur le droit de punir: on retrouve ici un aspect de la «question sociale pénalisée». Le souci de prévention, de «resocialisation» s'affirme avec le développement intensif des réflexions sur le patronage ou la protection de l'enfance ${ }^{7}$. On vise à «la veille et au lendemain de la prison $»^{8}$.

Un matériau existant et relativement fiable ${ }^{9}$ a permis de consacrer en un premier temps l'essentiel du travail aux membres de cette Société ${ }^{10}$. Travailler sur les hommes ne consiste pas à revenir à une histoire biographique classique $^{11}$ ni à une sociologie des acteurs «pure et dure», mais bien à utiliser le matériau biographique dans son accumulation afin de saisir, dans des itinéraires personnels, les points possibles de convergence, en quelque sorte traquer l'indicible. Les liens implicites qui apparaîtront seront regardés ici comme des éléments pertinents pour une compréhension globale des itinéraires politiques. Il s'agit donc d'une contribution à l'histoire politique qui ne tente pas d'oublier le projet, l'idéologie et la pratique, mais s'arrête d'abord sur les hommes, initiateurs, militants et adhérents, car leur formation, ou leur(s) attachement(s) pourrait expliquer ou préciser le sens d'un projet, au-delà de ce qu'il affiche. Et si ce seul travail sur les hommes ne peut totalement expliquer la genèse des politiques, il nous semble nécessaire à la compréhension de celles-ci.

On pourra observer la diversité professionnelle, politique, spirituelle d'hommes qui se retrouvent et travaillent ensemble. Il s'agit dès lors de s'interroger sur ces lieux - clubs, sociétés ${ }^{12}$ - où se met en œuvre un certain engagement et voir dans quelle mesure ils peuvent remettre en cause une "logique classique» de l'adhésion en politique. Il s'agit de comprendre quels éléments ancrent la participation de ces hommes pluriels à cet espace associatif, au-delà d'un projet affiché comme consensuel - le projet de réforme pénitentiaire - dont on peut penser que son lien à la «sécurité publique et privée ${ }^{13}$ en fait plus que tout autre un lieu de la construction de la solidarité. Il s'agit, enfin, de tenter de comprendre ce qui fait de ces hommes des réformateurs sociaux, avec quelle spécificité, en présentant tout d'abord les fondateurs et responsables avant de s'intéresser à la composition de cette Société. 


\section{Fondateurs et responsables: l'esprit SGP}

Les fondateurs

La Société générale des prisons, qui a pour objet «de contribuer à l'amélioration du régime pénitentiaire et d'éclairer l'opinion publique dans l'intérêt de la sécurité publique et privée ${ }^{14}$, se réunit pour la première fois le 7 juin 1877 à la mairie du Premier arrondissement de Paris. La Société, comme l'explique Jules Dufaure, son premier président, dans l'un des discours d'ouverture, est issue de la loi sur l'emprisonnement cellulaire de 1875 :

«C'est de ces débats parlementaires qu'est née notre Société; ce sont des inspirations qui en étaient sorties qui ont dressé à nos honorables fondateurs l'idée de créer la Société actuelle. Ils avaient été membres de toutes les commissions de l'Assemblée Nationale, ils avaient concouru à tous ses travaux; ils avaient été pour une grande part dans son œuvre, et je ne m'étonne pas qu'ils aient senti le besoin de la continuer encore en organisant la Société actuelle. ${ }^{15}$

Ancien bâtonnier, sénateur, membre de l'Académie française, Dufaure ${ }^{16}$ est assisté de trois vice-présidents: A. Fourichon, sénateur et ancien ministre de la Marine $^{17}$, Célestin Bétolaud, bâtonnier de l'Ordre des avocats à la Cour d'appel de Paris ${ }^{18}$ et René Bérenger, sénateur et, entre autres, vice-président du Conseil supérieur des prisons ${ }^{19}$. Un Conseil de direction de vingt personnes, six secrétaires, un secrétaire général et un trésorier composent cette première assemblée dont est absent Charles Lucas, pourtant père spirituel de la Société et de la réforme pénitentiaire. Je ne donnerai ici qu'un parfum de ce conseil fondateur, rassemblant députés et sénateurs, avocats, pasteurs, abbés, hommes du Conseil d'État, conseillers à la Cour de cassation, membres du Conseil supérieur des prisons, universitaires. Il est à l'image de la Société en 1877, une Société très investie par les professionnels et les élus politiques.

Ces hommes sont plutôt des libéraux, très liés aux régimes précédents, tels J. Dufaure ou A. Fourichon. Beaucoup sont des républicains libéraux qui appartiennent ou sont proches du groupe Feray, mais O. d'Haussonville est un orléaniste convaincu, catholique libéral ${ }^{20}$. Hommes déjà mûrs et âgés pour certains, hommes d'ordre et de morale, beaucoup d'entre eux appartiennent ou appartiendront à l'Académie des sciences morales et politiques ${ }^{21}$. voir F. X. Merrien, «État et Politiques sociales. Contribution à une théorie néo-institutionnelle ", Sociologie du travail, $\mathrm{n}^{\circ} 3.1990$, pp. 283-284.

13. Voir Charles Lucas, RP, 1877, p. 15.

14. Article II des statuts de l'association.

15. Jules Dufaure, «Discours d'audience ", $R P, 1877$, p. 41.

16. Voir «M. Dufaure», $R P, 1881$, pp. 576-580; «M. Dufaure», in Les magistrats et les avocats. Biographie des célébrités de la magistrature el du barreau, 1877-1878, pp. 83-95; Dufaure (1798-1881) in Richard Allou, Charles Chenu, Grands avocats du siècle, Paris, Pedone, 1894, pp. 104-123.

17. «M. Fourichon, Nécrologie», $R P, 1884$, pp. 843-844.

18. «M. Bétolaud». Notice ASMP (Académie des Sciences Morales et Politiques) 1906-1907, p. 76; voir Georges Dubois, « M. le bâtonnier Bétolaud". RP, 1915, pp. 357-359; R. Allou, Ch. Chenu, «Bétolaud», in Grands avocats du siècle, Paris, Pedone 1894, pp. 322-338.

19. Voir Charles Morizot-Thibault, «M. R. Bérenger», RP, 1915 , pp. 676-682; Charles Lyon-Caen, «René Bérenger 1830-1915», RP, 1924, pp. 30-39; Bernard Schnapper, «Le sénateur René Bérenger et les progrès de la répression pénale en France, 1870-1914", Annales Faculté de Droit, Istanbul, n ${ }^{\circ} 42,1979$, pp. 225-251.

20. Voir G. Weill, Histoire du catholicisme libéral en France, 1828-1908, coll. Ressources, 1935, rééd., Paris Genève Slatkine, 1979, p. 221.

21. Bérenger, Bétolaud, Desjardins, d'Haussonville, Picot, Lefébure, Renault, Ribot, etc. 


\section{O S S I E R}

Etatisations

Martine Kaluszynski

Réformer la société

22. Voir M. Kaluszynski, F. Tétard,

S. Dupont : «Un objet : l'enfant en danger moral, une expérience: la société de patronage ". Contrat MIRE-CNRS

«Fractures du social», 1990, 223 p.

23. Voir O. d'Haussonville, L. Lefébure, G. Picot. Sur G. Picot voir S. Magri, «Les laboratoires de la réforme de l'habitation populaire en France. De la SFHBM à la section d'hygiène urbaine et rurale du musée social, 1889-1909», Recherche, $n^{\circ} 72$, Plan, Construction Architecture, 1996; G. Le Béguec, «Les élites économiques et la naissance des formations politiques organisées, l'exemple des républicains modérés", in Guillaume (S.) (éd.), Elites fin de siècles $X I X^{e}-X X^{e}$ siècles, MSH Aquitaine, 1992, pp. 148-149; notice ASMP 1906-1907, pp. 149-160; L. Rivière, «M. G. Picot », BSGP 1909, pp. 1051-1059.
Ils peuvent être également considérés comme des «précurseurs » dans les domaines politique et social, à en juger par les liens, même ténus, avec la Société d'économie politique ou la Société d'économie sociale et par l'investissement concret de ces hommes dans la création des sociétés de patronage, institutions post-pénales en faveur des prisonniers libérés ou des enfants en danger moral ${ }^{22}$. Pour certains d'entre eux, fondateurs présents dès 1877 et véritables «piliers» de la Société, la préoccupation pénale n'est qu'une facette d'une préoccupation sociale plus large comme le montre entre autres leur rôle majeur dans le domaine de l'assistance ${ }^{23}$. Hommes de convictions, attachés à la religion - majoritairement ce sont plutôt des catholiques ou des protestants affirmés - ils sont aussi des républicains sincères, à la façon définie par d'Haussonville : «C'est le régime qui divise le moins et qui semble le plus propice à éloigner le spectre de la révolution.»

\section{Les responsables (1877-1914)}

Sur les hommes qui exercent des fonctions de responsabilité au sein de la SGP, seuls de grands traits pourront être ébauchés pour une période qui voit des changements de profils et d'enjeux. Le Conseil de direction, semblable en son organisation au Conseil fondateur de 1877, est à observer précisément. Des hommes y sont présents durablement qui, sans jamais tenir de rôle honorifique, auront un rôle moteur (le pasteur Arboux, P. Baillière, Bogelot, Chaix, E. Pages, E. Passez, notamment). Parmi eux, le secrétaire général, véritable cheville ouvrière de la Société, qui détient un vrai pouvoir sur l'organisation des séances, l'entrée de nouveaux membres et les finances. De 1877 à 1891, il s'agit de Fernand Desportes, un avocat défendant les causes civiles qui a été secrétaire de la conférence du stage en 1856-1857 et président de la conférence Molé, membre du Conseil supérieur des prisons et de la Société d'économie charitable de 1862 à 1877.

Si l'on s'attache, par facilité, aux postes honorifiques et néanmoins emblématiques de la Société et de son évolution, la liste des présidents et vice-présidents de 1877 à 1914 donne à voir, mieux que tout commentaire, et synthétise, par son panachage de noms, «l'esprit SGP». Ainsi les itinéraires d'un René Bérenger et d'un Henri Barboux surprennent et offrent des repères sur ce qui fut à un moment leur «destinée» commune de président ou vice-président. Entre Bérenger qui affirme à la tribune du Sénat: «Je suis 
un ferme républicain tout en étant conservateur et j'entends être et rester un très ferme catholique ${ }^{24}$ et Barboux qui présidera à la fondation de l'Union libérale républicaine, «polytechnicien en toge ${ }^{25}$, «dépourvu d'ambitions électorales et parlementaires» mais actif à la mise en place de «proto-partis $»^{26}$, il y a de nombreuses différences et de vrais points communs qu'on retrouverait chez la plupart des présidents et vice-présidents de la SGP.

Lettrés, érudits, beaucoup parmi eux sont juristes, plutôt avocats et en général brillants - plusieurs sont secrétaires de la Conférence du stage ou de la Conférence Molé. Quelle que soit leur religion, ces hommes y sont attachés et leurs principes reposent sur une conception de l'ordre social elle-même fondée sur le religieux. D'où, peut-être, un sentiment du devoir social qui passe par une restauration d'un ordre moral, à la fois sur un plan théorique et sur un plan pratique, comme en témoignent d'une part leurs liens avec l'Académie des sciences morales et politiques et, d'autre part, leur appartenance à la Ligue contre la licence des rues ou à la Ligue nationale contre l'alcoolisme.

Question pénale, question sociale se joignent pour ne faire plus qu'une et trouvent dans un "paternalisme juridique», selon l'expression chère à $B$. Schnapper ${ }^{27}$, une solution qui résiderait dans l'action combinée de l'État, de la famille et de tous les organismes sociaux. De J. Simon à E. Cheysson, l'idée apparemment «exhibée» serait celle d'une alliance à construire entre initiative publique et initiative privée, ce que nous montre Cheysson, cité par Boutroux lors de ses funérailles:

« Pourquoi se représenter dans ce domaine, l'État et la liberté comme deux puissances ennemies, dont l'un doit nécessairement expulser l'autre? Rien ne saurait remplacer les initiatives hardies, généreuses et fortifiantes de la liberté. D'autre part, sans qu'il entreprenne sur les libertés légitimes, il reste à l'État en matière sociale maintes attributions pratiquement utiles ou nécessaires, que l'on aurait tort de lui disputer. ${ }^{28}$

Ces hommes sont des républicains... modérés, des libéraux... interventionnistes, des démocrates... sans excès. $\mathrm{Ni}$ chefs de parti, ni directeurs d'affaires du pays, ils allient les traditions paternelles d'indépendance et de libéralisme aux vertus du travail et de la science. Ils ont une conception pratique et méthodique du monde social. $\mathrm{Ni}$ doctrinaires, ni romantiques, pour la plupart d'entre eux revient l'expression d'hommes de «juste milieu». À
24. Voir Ch. Morizot-Thibault, «R. Bérenger », Revue pénitentiaire, 1915, pp. 675-676.

25. Selon l'expression de Gilles Le Béguec, «L'aristocratie du barreau, vivier pour la République. Les secrétaires de la conférence du stage ", Vingtième siècle, avril-juin 1991, p. 23.

26. Voir G. Le Béguec, «Les avocats et la naissance des partis politiques organisés (1888-1903)", Histoire de la Justice, $\mathrm{n}^{\circ}$ 5, 1992, pp. 171-188, (Sur H. Barboux, pp. 181-182). Sur H. Barboux voir G. Le Béguec, Les élites économiques..., op. cit, 1992; Notice BSGP 1910, pp. 613-614; F. Masson, «Discours pour les funérailles d'H. Barboux, 27 avril 1910», Institut - Académie française, pp. 1-10; R. Allou, Ch. Chenu, Grands avocats du siècle, Paris, 1894, pp. 340-361.

27. Cette notion traverse de nombreux travaux de B. Schnapper. Il l'a utilisée bien souvent lors de ses interventions orales et c'est de cette manière que nous lui empruntons ainsi cette formule.

28. Voir E. Boutroux, Discours prononcé aux «Funérailles d'E. Cheysson ", Institut-Académie française, 1910, p. 5. Sur E. Cheysson: A. Desrosières, «L'Ingénieur delta et le père de famille, E. Cheysson et la Statistique ", Annales des Mines, Série Gérer et Comprendre, 1986, n² 2 , pp. 66-80; S. Laurent, «E. Cheysson (1836-1910). Entre modernisme et paix sociale. Portrait d'un Le Playsien modèle », Actes du $117^{\mathrm{e}}$ Congrès national des Sociétés Savantes, Clermont-Ferrand, 1992, Paris, Association pour l'étude de l'histoire de la Sécurité sociale, 1994, pp. 131-145. 


\section{O S S I E R}

Etatisations

Martine Kaluszynski

Réformer la société égale distance du despotisme et du radicalisme, ils croient à l'influence salutaire des idées libérales alliées au sentiment du bon ordre pour prévenir les violences de l'esprit révolutionnaire.

\section{Les hommes de la SGP, des réformateurs sociaux?}

La Société générale des prisons, de par ses objectifs, son organisation, un certain mode de fonctionnement, de par la diversité et l'éminence de ses membres doit-elle être prise comme «idéal-type» du projet et de l'espace réformateur, tel que nous cherchons à l'appréhender? Projet réformateur égale-t-il espace réformateur, espace réformateur égale-t-il esprit réformateur? Si cette adéquation est exacte, à observer les hommes qui sont à la tête de la SGP, hommes d'ordre et de morale, ne faut-il pas considérer que les "réformateurs" seraient proches des «conservateurs»? Ne faut-il pas s'interroger sur l'image implicite que nous avons de la réforme sociale et de ses lieux?

Notre premier sentiment sur ce que serait la formation d'un espace réformateur se fonde sur sa capacité à recueillir une adhésion minimum sur un projet commun, consensuel, d'hommes aux engagements divers. À partir de cette hypothèse nous tenterons de déceler comment se forme l'adhésion et nous nous interrogerons sur la qualité des membres de la SGP à être reconnus réformateurs. Mais tout d'abord, que nous montre la composition de cette Société?

Parmi ses nombreux membres - 1500 adhésions sont enregistrées de 1877 à 1900 - toutes les professions libérales sont représentées mais les juristes sont les plus nombreux. Sans énumérer toutes les fonctions possibles de ceux-ci dans la "gestion de la justice et des conflits", notons le rôle des universitaires. Les professeurs de droit sont en nombre et l'on y retrouve beaucoup de théoriciens, porteurs d'une philosophie pénale nouvelle ou différente. Cet investissement juridique se comprend si l'on considère le projet initial de la SGP qui a pour objet la réforme pénitentiaire et pénale. Ajoutons que la plupart de ces hommes sont liés à l'Académie des sciences morales et politiques. Ici encore, la conviction, sans doute, que la question pénale et sa résolution sont liées à la nécessité d'une action morale qui est au fondement de toute initiative pouvant régler la question sociale. 
Un espace de convictions:

$d u$ poids $d u$ religieux et du politique

La Société est ouverte aux ecclésiastiques de toutes confessions $^{29}$. Cette observation permet de préciser l'image de ces hommes qui, bien qu'attachés à la République, ne sont pas des anticléricaux notoires et restent proches de l'esprit insufflé par leurs origines confessionnelles. Certains pourront publiquement s'en démarquer, d'autres non ${ }^{30}$, mais aucun de ces hommes n'exclura réellement son appartenance religieuse de son itinéraire et de sa pratique, et beaucoup s'en inspireront dans un projet de réforme pénitentiaire imprégné de l'idée d'amendement. On retrouve ici l'abbé Crozes, le pasteur Arboux, connu également pour son implication dans les questions de la mutualité, ou les pasteurs Robin ou Rouville, sans oublier Zadoc Kahn, grand rabbin de France, connu pour son activité religieuse militante et philanthropique ${ }^{31}$.

Cette société para-administrative est, très tôt, fortement investie par les hommes politiques mandatés. De 1877 à 1900 , toutes les tendances sont présentes, sans grand extrémisme: des républicains conservateurs (A. André, G. Balsan), de la Gauche démocratique (A. Bérard, D. Folleville, L. L. Klotz, L. Lépine, P. Strauss), de l'Union républicaine (E. Flandin, H. Barboux, Ferdinand-Dreyfus, V. Schoelcher) à la Gauche radicale (J. Cruppi, H. Cornudet). Au sein de la SGP des positions-clés ont été tenues par beaucoup d'hommes de l'Union libérale républicaine sans qu'on puisse dire pour autant qu'il y ait connivence entre les deux lieux ${ }^{32}$. La période observée, bien que restreinte, marque déjà des changements, des effets de génération, des évolutions de projets, de philosophie, de sensibilités. 1895 est un moment qui semble marquer un changement au sein de la SGP, en particulier quant à «l'esprit» politique qui traverse la société à partir de ses élus ${ }^{33}$. Même s'il reste des piliers inébranlables qui, de 1877 à 1900, structurent la Société (Ferdinand-Dreyfus, R. Folleville, L. Lefébure, O. d'Haussonville, A. Ribot, T. Roussel, F. Voisin et le sénateur R. Bérenger), certains ont disparu, même parmi les fidèles: A. André, A. Desjardins, F. Chauveau, tandis qu'une nouvelle "génération", dès 1895 , semble apparaître parmi les députés: L. L. Klotz, G. Dubois, J. Leveille, J. Cruppi et parmi les sénateurs: E. Marvejouls, G. de Normandie, P. Strauss.
29. On dénombre 23 rabbins, 15 pasteurs, 24 prêtres ou évêques de 1877 à 1900 à l'intérieur de la SGP.

30. Entre autres H. Joly, qui est vice-président de la Ligue contre l'athéisme et fervent catholique.

31. Voir P. Birnbaum, Les Fous de la République, Paris, Fayard, 1992, pp. 88-89 et de nombreuses pages disséminées dans R.M. Marrus, Les Juifs de France à l'époque de l'affaire Dreyfus, Coll. Diaspora, Calmann Lévy.

32. Voir G. Le Béguec, «Les avocats et la naissance des partis politiques organisés, 1883-1903 ", Histoire de la Justice, $\mathrm{n}^{\circ} 5,1992$, pp. 171-188 (G. Picot, H. Barboux, Leredu, P. Boncour...)

33. D'où la nécessité, sur cette question (entre autres), d'avancer le dépouillement au-delà de 1900 pour saisir sur le long terme ces changements, que cinq ans seulement (1895 à 1900) ne suffisent pas à analyser avec finesse. 
D O S S I E R

Etatisations

Martine Kaluszynski Réformer la société
Un espace associatif: du poids de la société civile

La SGP serait ainsi un espace à la fois professionnel et politique, montrant dès lors les caractères saillants d'un projet alliant compétence et pouvoir. Elle s'impose également comme un lieu ouvert, fidèle à l'esprit du projet fondateur qui l'anime: la mobilisation du plus grand nombre sur les questions sociales. L'éclectisme et la diversité des hommes de cette Société en font un espace mélangé, permettant la mixité sous toutes ses formes, non seulement sexuelle, nous le verrons, mais également professionnelle, politique, identitaire. Républicains modérés, laïques, catholiques convaincus, francs-maçons reconnus, se côtoient, mêlant la revendication de l'initiative privée à un attachement à l'engagement de l'État tentant de mettre en œuvre la réforme pénitentiaire.

Serait-ce donc cela des réformateurs sociaux? Un rassemblement d'hommes «de bonne volonté», d'origines différentes, portés par un même objectif, montrant leur capacité à l'efficacité. Tentons de cerner comment se réalise cette adhésion multiforme, s'établit ce relatif «consensus» qui unit, soude et rapproche ces hommes épars, venant de tous les pays, de toutes les régions de France. Cette adhésion qui est peut-être un des éléments dans lequel la réforme sociale trouve son unité.

\section{Des conditions du consensus}

L'observation des règles de fonctionnement, des modes d'organisation, des méthodes de travail et de publicité de la SGP permet de repérer certains processus essentiels à la formation de la cohésion du groupe et à sa réussite.

\section{Le contrôle}

Cet espace repose sur l'esprit associatif, le bénévolat, le libre choix, l'ouverture à tous, enfin à presque tous car il faut être parrainé par deux membres agréés par le Conseil de direction pour devenir membre titulaire. Un rituel extrêmement codifié précède l'adhésion. C'est donc sous les signes de la rigueur, de l'assiduité et du passage ritualisé que la Société générale des prisons s'ouvre à ses membres. Là, chacun peut trouver ou peut prendre sa place dans un espace contrôlé.

\section{L'organisation}

Les statuts prévoient un président élu pour deux ans et non immédiatement rééligible, quatre vice-présidents, un 
secrétaire général, quatre secrétaires au minimum, nommés chaque année par un Conseil de direction de vingt membres au moins élus pour quatre ans renouvelables par quart et non immédiatement rééligibles. Il existe également deux commissions nommées par le Conseil de direction: la Commission des études, qui s'occupe du Bulletin et de l'ordre du jour, et la Commission des œuvres, qui s'occupe des activités externes. Enfin, des séances mensuelles réunissent de décembre à juin tous les sociétaires sur des sujets d'études mis à l'ordre du jour par le Conseil de direction.

La Société est divisée par ailleurs en quatre sections correspondant aux diverses branches de ses travaux: «Législation pénitentiaire en France», «Du régime pénitentiaire en France et du patronage des adultes», "De l'éducation correctionnelle, du patronage des jeunes libérés et des mesures préventives», «Des questions pénitentiaires à l'étranger». Présidées par les vice-présidents, les sections sont composées de tous les membres qui demandent à y être inscrits. En 1886, les quatre sections se recomposent en trois. Les anciennes deuxième et troisième sections «fusionnent» pour être consacrées au «Patronage et mesures préventives». Cet effort synthétique correspond au cheminement des hommes de la SGP qui, dès lors, vont concentrer leurs efforts sur les questions de prévention.

\section{La diffusion des idées}

Quelles sont les armes qui permettent à une société d'être offensive? Ce sont: les ressources, les finances ${ }^{34}$ et les instruments qui permettent à un «mouvement» d'exister et de diffuser ses idées. Parmi ceux-là, une revue et les congrès internationaux jouent un rôle essentiel.

L'organe de la Société s'intitule Bulletin de la Société générale des prisons jusqu'en 1892 et prend ensuite le titre de Revue pénitentiaire et de droit pénal. Ce Bulletin a la même périodicité que les séances générales: il en reproduit les procès verbaux, il contient les rapports déposés dans chacune d'elles, les travaux des sections et de ceux des membres qui ne sont pas de nature à être l'objet d'une discussion orale, les documents et les communications recueillis par le conseil, enfin sous la forme de Revue, l'exposé des faits et l'analyse de livres utiles à connaître pour l'œuvre pénitentiaire.

Le Bulletin comprend aussi des informations diverses: nominations, décrets, circulaires, nouvelles, notices nécro-
34. Ici même, ce sont les cotisations, les souscriptions, les dons et legs, les subventions qui assurent les finances de la Société. De plus, les fonds disponibles sont placés en rentes nominatives, 3 \% sur État, et en obligations nominatives de chemin de fer dont le minimum d'intérêt est garanti par l'État. 


\section{O S S I E R}

\section{Étatisations}

Martine Kaluszynski Réformer la société

35. 1872 Londres, 1878 Stockholm, 1885 Rome, 1890 Saint-Pétersbourg, 1895 Paris, 1900 Bruxelles, 1905 Budapest, 1910 Washington, 1925 Londres, 1930 Prague, 1935 Berlin, 1950 La Haye.

36. Voir revue Mil Neuf Cent, revue d'histoire intellectuelle. Les congrès, lieux de l'échange intellectuel, 1850-1914, 7-1989, notre article sur « Les congrès internationaux d'anthropologie criminelle, 1885-1914", pp. $59-76$.

37. Charles Daru, Victor Bournat, «La Société royale des prisons. $1819-1830$ », RP, 1879 , pp. 54-72, pp. 288-301, pp. $441-464$, pp. $729-751$. logiques. Il est distribué à tous les membres de la Société, tant en France qu'à l'étranger, la réception du Bulletin étant liée au statut de membre. C'est la librairie centrale des chemins de fer, A. Chaix et Cie, qui est chargée de la vente et du service des abonnements à l'étranger.

S'il n'y a pas de congrès national sur le thème, de nombreux congrès sur les patronages réuniront les hommes de la réforme pénitentiaire. En outre, des congrès pénitentiaires internationaux, au nombre de douze entre 1872 et $1950^{35}$, permettront de structurer le mouvement à la fois sur le terrain national et international ${ }^{36}$.

\section{L'ancrage: tradition et attachement}

L'organisation de la Société, telle que ses statuts la prévoient, ne laisse rien deviner de la réalité de son fonctionnement. Il est difficile d'évaluer la participation effective, l'assiduité des membres au travail de la Société. Les indicateurs de participation que l'on peut prendre sont l'appartenance à un Conseil de direction, la fréquence des signatures au bas des articles publiés dans le Bulletin, les interventions lors des séances. Si l'on additionne ces données, on obtient entre 150 et 200 personnes. Par ailleurs, l'observation de l'adhésion à long terme des sociétaires permet de présumer une réelle «implication» de leur part. Les «piliers», présents de 1877 à 1900, représentent à peu près 120 personnes et les «fidèles ", présents de 1877 à 1895 ou de 1884 à 1900 , sont autour d'une centaine de personnes. Voilà donc près de 230 personnes auxquelles on peut reconnaître un intérêt "durable» pour la SGP et son projet.

Cette présence assure une stabilité, une structuration à un mouvement qui peut compter sur cet ancrage humain important, allié peut-être à l'ancrage historique à cette Société républicaine, héritière de la Société royale des prisons créée en 1819. Emportée par la Révolution de 1830, la Société royale disparaissait en même temps que le régime de la Restauration. En 1847, à la Chambre des Pairs, Bérenger (de la Drôme), rapporteur de la commission chargée d'examiner le projet de la réforme pénitentiaire présenté par le gouvernement, constatait les grands services rendus par cette Société, regrettait sa disparition et formulait des vœux pour son rétablissement ${ }^{37}$. Ce souhait fut réalisé trente ans plus tard et la SGP hérita de cet acquis intellectuel, politique et organisationnel. Ancrage historique, 
ancrage humain, investissement personnel et concret concourent à la cohésion du groupe, cohésion renforcée par les liens du travail.

\section{Les chantiers intellectuels}

La SGP est aussi un lieu d'études organisées méthodiquement. Enquêtes et questionnaires sont délivrés à toutes personnes susceptibles de répondre. En 1879, par exemple, un questionnaire concernant les prisons cellulaires et les dépenses nécessaires à leur construction est établi. La SGP reçoit des réponses en provenance de Belgique, du Danemark, de Hollande, de Suède, d'Allemagne, d'Autriche, de Suisse et de Grande-Bretagne.

Méthode et savoir-faire empruntent au positivisme ambiant. Ch. Lucas dans son rapport à l'Académie des sciences morales et politiques en 1879 écrivait:

«La Société des prisons en procédant par des enquêtes est entrée dans la bonne voie, dans la véritable méthode scientifique, celle de l'observation pratique. On ne saurait trop lui conseiller de ne jamais s'en départir. C'est ainsi qu'elle travaille à la création de la science pénitentiaire par les deux conditions essentielles qui peuvent seules y conduire, l'expérience pratique d'abord et ensuite l'étude méditative. ${ }^{38}$

Nous observons ici la réalité d'un espace de travail où les professionnels exercent leurs compétences, les érudits aiguisent leur curiosité et où, toujours, de la confrontation, émergent des réflexions qui permettront d'échafauder ce qui deviendra «la science pénitentiaire».

Ainsi, à s'interroger sur ce qui apparaît une réussite liée au bon fonctionnement de la Société, quelques réponses apparaissent: un espace contrôlé, organisé, un espace doté d'instruments de diffusion, un lieu investi, ancré humainement et traditionnellement, un projet associatif fondé sur le travail, l'expertise, où la science, vertu consensuelle, réunit des hommes de nationalités ${ }^{39}$ et d'opinions différentes - comme l'indique bien Lucas en 1877 dans un discours d'ouverture:

" "Quand les temps sont nébuleux", [c'est] un motif de plus pour se rapprocher de cette région sereine de la science [...]. C'est là pour les hommes de tous les partis un terrain neutre sur lequel ils peuvent s'unir et travailler en commun à la solution de ces grands problèmes qui ont tant d'attraction pour tous $[\ldots])^{40}$

Qualité pacificatrice de la «méthode» mise en œuvre, savoir-faire unificateur, compétence, "professionnalisme», projet mobilisateur sont autant d'éléments qui for-
38. Ch. Lucas, «Rapport à l'A.S.M.P., 1879 », séance 19 avril $1879, R P, 1879$, pp. 697-698.

39. Le Comte Sollohub, Conseiller de S.M. L'empereur de Russie écrivait : «Je suis étranger parmi vous mais je crois que les hommes de la même science sont toujours compatriotes. Le bien de l'humanité est la patrie universelle». « Rapport sur la répression de la récidive en Russie », $R P, 1877$, p. 45.

40. Lucas, Discours d'ouverture, $R P, 1877$, p. 14. 
D O S I E R

Etatisations

Martine Kaluszynski Réformer la société

41. Voir M. Trebisch, N. Racine (éds), Sociabilités intellectuelles. Lieux, milieux, réseaux, Cahiers $I H T P$ n $^{\circ} 20$, 1992.

42. Toutes les données qui suivent concernent la période 1880-1914 dans son ensemble. gent une idéologie «douce» où se retrouvent à la fois le respect des exigences méthodologiques et déontologiques, contribuant à l'adhésion du plus grand nombre dans un espace où les logiques peuvent être plurielles, les choix multiples. Dès lors, les réformateurs sociaux ne seraientils pas ceux qui sont capables de s'unir sur un projet et, audelà de celui-ci, de transcender les clivages par leur activité - qui n'est pas forcément réformatrice par nature - et de mettre en œuvre du débat, voire de l'action?

\section{Réformateurs sociaux, nébuleuse réformatrice: une construction de l'objet}

L'image du réformateur social, et surtout de la nébuleuse réformatrice, n'est-elle pas liée à cette observation: l'appartenance multiple d'hommes à différents espaces, mais qui partagent des objectifs similaires en l'esprit. Dès lors la nébuleuse réformatrice ne devient-elle pas la "constellation" obligée dans le paysage intellectuel, authentifiant la qualité de celui que nous cherchons à cerner avec difficulté, le réformateur social?

De quoi serait-elle constituée, cette nébuleuse? N'y a-t-il pas un choix subjectif propre à chacun de nous d'y intégrer un lieu plutôt qu'un autre mais, à l'inverse, ces hommes «éclatés», incontournables, ne viennent-ils pas, par leur présence à un endroit et leur ubiquité déroutante dans le domaine social et politique, valider un espace comme réformateur alors que la question reste toujours posée? L'hypothèse d'une nébuleuse réformatrice met en place l'idée d'une dynamique de réseaux ${ }^{41}$ qui constituerait l'édifice réformateur. Mais n'évacue-telle pas du même coup la possibilité d'une définition plus précise, plus aiguë, du réformateur social? Il est vrai que cette hypothèse semble pertinente au sein même de la SGP: à travers ses membres, on repère de multiples attaches qui feraient de celle-ci un «réseau», un maillon du «réseau» réformateur traversé lui-même par d'autres « réseaux».

Cette accumulation d'appartenances aurait pouvoir à renforcer, à augmenter subtilement, tel un ciment invisible, la force sociale, la "position", le pouvoir des hommes à l'intersection de différents espaces. Ce lien informel a ses repères et se construit selon des filiations différentes. Nous en avons repéré quelques-unes en ce qui concerne la SGP ${ }^{42}$. 
Assez logiquement, beaucoup de membres de la SGP appartiennent au Conseil supérieur des prisons, organisme consultatif institué dès 1875 auprès du ministre de l'Intérieur: on en compte environ quarante au cours de la période 1880-1914. Les liens sont nombreux avec la Société de législation comparée - à laquelle appartiennent notamment G. Picot, A. Poittevin, F. Daguin, A. Dareste, L. Aucoc et C. Lyon-Caen - et avec la Société d'études législatives: non seulement $\mathrm{H}$. Barboux, $\mathrm{H}$. Berthélémy, G. Picot et F. Daguin appartiennent à celle-ci, mais des séances mixtes sont tenues avec la SGP en juin 1903 et juin 1922.

Les membres de la Société d'économie sociale ${ }^{43}$ ne sont pas rares à la SGP: c'est le cas par exemple d'E. Cheysson, A. Gigot, J. Lacointa, L. Lefébure et G. Picot, H. Joly (président 1905), etc. En 1914, $\mathrm{P}$. Nourrisson, A Rivière, $\mathrm{H}$. Joly et $\mathrm{L}$. Rivière siègent au Conseil d'administration de la société des disciples de F. Le Play. Par ailleurs, J. Simon, A. Ribot et H. Barboux appartiennent au Musée social ${ }^{44}$, le dernier en dirigeant la section juridique, G. Picot assurera la responsabilité de la section «institutions patronales» et la section «missions».

Avec le pasteur Arboux, C. Robert, A. Gigot ou H. Marès, la SGP est liée au mouvement mutualiste, mais elle est plus présente encore dans le domaine de l'assistance et de la bienfaisance: L. Lefébure, G. Picot, F. Voisin et $H$. Thulié appartiennent à l'Office central des œuvres de bienfaisance - et, en outre, Guérin Pélissier à celui du Nord - tandis que L. Brueyre, H. Thulié et $\mathrm{E}$. Cheysson entrent tous trois au Conseil supérieur de l'assistance publique entre 1891 et 1897 . Pour ce qui est des activités de terrain, les sociétés de patronage comptent une cinquantaine de membres de la SGP parmi leurs dirigeants. Plus ciblées, la Ligue nationale contre l'alcoolisme ou la Ligue contre la licence des rues réunissent également quelques-uns de nos sociétaires.

De nombreux membres de la SGP soutiennent financièrement l'École libre des sciences politiques - certains souscrivent à son capital dès $1872^{45}$ - ou y enseignent: ainsi A. André, L. Aucoc, E. Glasson, C. Lyon-Caen, L. Renault, A. Ribot, L. Lefébure, A. Mirabaud, A. Rousse, P. de Remusat. L'Alliance française aura A. Ribot, A. Rousse, J. Simon, O. d'Haussonville parmi ses membres d'honneur, Ferdinand-Dreyfus et Zadoc
43. Sur la SES et le mouvement leplaysien, voir B. Kalaora. A. Savoye, Les inventeurs oubliés. Le Play et ses continuateurs aux origines des sciences sociales, Seyssel-Champ Vallon, 1989, et «Les réformateurs sociaux en France et en Italie 1889-1914 ", Études sociales, n ${ }^{\circ} 118$, 1989 , pp. 45-76.

44. Voir Janet R. Horne, Republican social Reform in France. The Case of the Musée social, 1894-1914,

Ph. D., New York University, 1992.

45. Liste officielle des actionnaires de l'ELSP, Archives FNSP, ISP 43-dr 5.1. Voir la thèse de $R$. Vaneuville, en cours d'achèvement, sur la représentation de l'Angleterre à l'École libre des sciences politiques de 1871 à 1914; et D. Damamme, "Genèse d'une institution scolaire. L'École libre des sciences politiques". Actes de la recherche en sciences sociales, $\mathrm{n}^{\circ} 70$, 1987, pp. 31-46. 


\section{O S S I E R}

Etatisations

Martine Kaluszynski Réformer la société

46. P. Foncin, L'Alliance française. Mémoires et documents publiés par le Musée pédagogique, Paris. Imprimerie nationale, 1889.

Voir Marie-Angela Roselli, «Les hommes de l'Alliance française, 1883-1889. Entre Université et politique», chapitre de sa thèse La langue française entre science et république, 1880-1950, IEP Grenoble, 1994, 667 p.

47. Voir J.-G. Petit, Ces peines obscures [...], op. cit., pp. 594-595.

48. Jacqueline Lalouette conteste cette information mais admet $\mathrm{V}$. Schoelcher dans le mouvement de la Libre pensée. Voir Jacqueline Lalouette, "Sous l'égide de la libre pensée ", in (sd) J.-M. Mayeur, A. Corbin (éds), Les immortels du Sénat, 1875-1918, Publ. Sorbonne, 1995.

49. Voir P. Chevalier, Histoire de la franc-maçonnerie française, tome 5., voir anonyme, Liste des membres de la franc-maçonnerie, Lyon, Ain, Rhône, Isère, 1895, BN ( $8^{\circ}$ M1566); voir M. Gaudart de Soulages et H. Lamant, Dictionnaire des Francsmaçons, Paris, Albatros, 1980.
Kahn parmi ceux de son Conseil d'administration ${ }^{46}$. L. Herbette, directeur de l'Administration pénitentiaire et membre actif de la SGP, en sera l'un des vice-présidents ${ }^{47}$.

Très nombreux sont les membres de la SGP dont la notabilité scientifique est consacrée par une élection à l'Institut: tantôt à l'Académie française - comme le duc de Broglie, J. Dufaure, J. Favre, le vicomte O. d'Haussonville, A. Rousse ou J. Simon - tantôt à l'Académie de médecine - les docteurs Bergeron, Bouley, Motet, Roger, Roussel et Wurtz - mais plus souvent encore à l'Académie des sciences morales et politiques: de 1868 à 1920 , on ne compte pas moins de trente-cinq membres de la SGP qui y siègent, parfois comme académiciens libres ou correspondants de l'Institut de France, le plus souvent comme membres des sections de Morale, de Législation, d'Économie politique ou de Philosophie. On retrouve Georges Picot, à la fonction de secrétaire perpétuel de l'Académie des sciences morales et politiques de 1896 à 1909, et J. Simon (1871), F. Passy (1890), L. Aucoc (1891), G. Picot (1892), E. Glasson (1897), A. Desjardins (1898), R. Bérenger (1903), C. Lyon-Caen (1905), A. Ribot (1915), H. Joly (1916), C. Morizot-Thibault (1919), H. Berthélémy (1930), occupent la fonction illustre de président de cette institution.

Des liens plus secrets peuvent traverser ces lieux, mais leur contribution à la construction de la nébuleuse réformatrice reste mal connue. Il est notoire que V. Schoelcher, membre de la SGP de 1877 à 1885, vice-président du Conseil supérieur des prisons en 1884, est franc-maçon ${ }^{48}$. Les sources existantes ne permettent pas de repérer facilement ses éventuels co-disciples. Une dizaine de membres de la SGP ont pu appartenir à la franc-maçonnerie - comme Chabrière-Arlès, A. Bérard, H. Thulié, P. Nourrisson, Chaumie, Cruppi, etc... ${ }^{49}$ : ce ne sont pas des responsables au sein de la Société mais des hommes actifs dans leur domaine. Ce thème reste à creuser, à la fois du point de vue de l'information et de celui de la connexion entre réseaux.

Enfin, il existe des filiations naturelles et liens de parenté entre divers membres de la SGP, où l'on voit apparaître la famille comme un réseau privilégié. Ainsi Choppin, le directeur de l'Administration pénitentiaire, membre de la SGP, est le beau-frère du bâtonnier Cresson, président de la Société. L'avocat $\mathrm{H}$. Tournouër et 
Albert Rivière sont les gendres du conseiller Félix Voi$\sin ^{50}$. Il y a des «dynasties» qui assurent leur présence et leur nom au sein de la Société de père en fils - les Flandin, les Rivière, les Yvernès - ou en couple: les Ferdinand-Dreyfus, les Mallet, les Desportes, ces derniers accompagnés d'autres membres encore de leur famille. Ici aussi, ces liens viennent cimenter une cohésion existante plus que solidifier, me semble-t-il, l'esprit réformateur.

Il y a sûrement beaucoup d'autres réseaux qui traversent la SGP. Cette rapide énumération montre simplement l'intense activité de nos sociétaires en ces domaines, les connexions susceptibles de se profiler, les lieux intéressants à "re-visiter ", le potentiel humain dynamique et mobilisé se façonnant des outils communs, cuvrant vers un même objectif quelles qu'en soient les formes, quels qu'en soient les termes: celui de la réforme sociale.

La recherche que nous avons entreprise sur les attaches multiples, consolide l'idée d'une "nébuleuse réformatrice». Celle-ci ne serait-elle pas une construction d'objet qui appréhende la réforme sociale dans une dimension de pouvoir et d'influence, une franc-maçonnerie invisible et efficace qui, de par sa nature même, ne peut-être cernée mais seulement imaginée? Il s'agirait donc d'un subtil jeu de passe-passe pour contourner la difficulté qu'il y a à définir, et donc à donner existence, à cet axe de pensée, à ce mouvement de la réforme sociale, dont le rôle nous semble pourtant déterminant dans la gestation d'expériences innovantes, voire dans la mise en ouvre des politiques. Cet entrecroisement d'hommes et de lieux vient enrichir et complexifier l'interrogation sur l'association comme lieu d'élaboration et de mise en œuvre d'un projet social et politique. Selon les hommes, ce projet penche tantôt du côté social, tantôt du côté politique, parfois se tient entre les deux, ce qui indique peut-être l'une des composantes de ce que serait l'esprit réformateur: une combinaison du social et du politique dont le dosage dépend de la sensibilité de chaque individu. Cette éventuelle définition ne nous permet toujours pas de comprendre la construction et l'identité du phénomène, et peut-être faut-il revenir au projet comme indicateur concret et sur les modalités mises en œuvre comme révélateur essentiel de cet engagement associatif, social et politique.
50. Voir L. Rivière, «Monsieur le conseiller Félix Voisin », RP 1915, p. 593 (A. Rivière), p. 598, note 2 (H. Tournouër). 


\section{O S S I E R}

Etatisations

Martine Kaluszynski

Réformer la société
51. «L'usage du désintéressement par la profession ne relève ni de l'angélisme, ni de l'irréductible singularité, il manifeste un mécanisme général que l'échange primitif fait apparaître dans sa plus grande pureté. La détermination fondamentale tient à la relation triangulaire entre un acteur collectif qui entend sauvegarder un mode d'existence (l'indépendance), des adversaires plus puissants (ici le marché et l'État) qui menacent de l'englober et de l'anéantir socialement dans une logique de fonctionnement qui lui est étrangère, un tiers parti (le public) dont la position détermine l'issue du conflit. " L. Karpik, «Une éthique professionnelle.

Le désintéressement ». Annales ESC, mai-juin $1989, n^{\circ} 3$, p. 745.

52. Madame Beaury Sorel, inspectrice des prisons de Saint-Lazare et inspectrice générale des prisons de la Seine en 1885, Madame Dupuy, inspectrice des services administratifs du ministère de l'Intérieur, Madame Opezzi de Cherio, inspectrice générale des prisons.

\section{L'appartenance à la SGP: les enjeux du projet réformateur}

Il est extrêmement délicat de repérer tout ce que peut amener «l'appartenance» à une association, et à celle-ci en particulier. On ne peut qu'émettre des hypothèses et esquisser les enjeux divers qui pourraient s'y profiler et dont certains forcément nous échappent, liés à des motivations enfouies au cœur de l'individu.

Des enjeux professionnels peuvent se dessiner dans ce lieu de la réforme pénitentiaire. Promotion sociale pour les agents de l'administration qui trouvent ici un moyen de renforcer des compétences professionnelles par une activité d'expertise et d'évoluer dans une sphère où se discutent des projets et se prend l'initiative de lois dont ils seront à la fois promoteurs et exécutants. Ils se constituent ainsi une légitimité supplémentaire dans un champ satellite. Les avocats peuvent trouver à la SGP un enjeu symbolique car leur activité y relève d'une certaine gratuité du geste et d'une idéologie du désintéressement que L. Karpik a justement évoquée en ce qui concerne les juristes, ces médiateurs par excellence exerçant une profession qui repose sur une valeur: la justice ${ }^{51}$.

Les femmes - 21 sont recensées de 1877 à 1900 - quelle que soit leur activité, trouvent ici un lieu d'émancipation, un lieu de transition parfait entre expérience pratique et phase théorique, en parallèle à une activité philanthropique classique. Présidentes d'œuvres de patronage, épouses de sociétaires éminents mais, également, professionnelles du monde pénitentiaire ${ }^{52}$ évoluent au sein de cette société.

La SGP peut être également un lieu d'initiation pour les jeunes hommes qui s'exercent aux postes de secrétaires et s'élèvent tout en renforçant leurs compétences et leurs statuts, mais aussi un lieu de consécration pour de plus âgés, aux profils différents, qui trouvent ici un moyen de continuer une activité ayant trait à de grandes questions sociales. C'est aussi, et peut-être principalement, un lieu de participation permettant, par ce processus d'engagement associatif fort, de construire en quelque sorte sa citoyenneté et d'accéder de façon privilégiée au débat public en élaborant des projets législatifs ayant trait aux questions de justice. En ce sens, la SGP est un lieu d'exercice du politique. On s'y instruit, on y discute, on se donne les moyens d'explorer les questions 
importantes, sans pour autant entrer dans les formes traditionnelles du politique.

Ceci se déroule toutefois dans un contexte particulier, car la période choisie ici pour observer la SGP, de 1877 à 1900 , est un temps de recomposition et même de bouleversement. 1877, la République opportuniste est au pouvoir et a engagé le processus parlementaire de sa transformation sans avoir de solution a priori. Il y a un changement de nature dans le traitement et l'appréhension de la question sociale. Comme l'explique F. Ewald: «Les conditions du discours solidariste se trouvent dans la philosophie politique des opportunistes. Ce sont eux qui ouvrent la possibilité de sa formulation et, dans la mesure où la seule obligation solidariste est une obligation de négocier en permanence les conditions du contrat social, on peut dire que les opportunistes inaugurent la modernité politique " ${ }^{53}$. Dans ce contexte, la SGP, espace mixte, intermédiaire entre l'État et la société, fonctionne comme un «réseau de médiation ${ }^{54}$ : officiellement espace associatif, proche néanmoins de l'image du «service public» du début du siècle, caractérisée par la volonté de confier la gestion de l'action publique à des professionnels compétents censés détenir les savoirs et les méthodes adaptés aux grands problèmes sociaux ${ }^{55}$.

Concertation, communication, informations donnent la souplesse et l'efficacité dont a besoin la Société pour fonctionner. Celle-ci est animée d'une logique du «juste milieu » ou de l'entre-deux qui neutralise les forces diverses en présence ${ }^{56}$, canalisant l'énergie et les potentiels. Action et surtout interaction favorisent l'échange, fondent la légitimité nécessaire à une certaine efficacité politique. Dans ce contexte, la SGP, dans son projet, n'est, ni ne peut être, doctrinale ou clivée. C'est dans des réponses pragmatiques, expérimentales qu'elle tente d'amener des solutions aux problèmes sociaux. L'idéologie ici ne peut avoir un rôle mobilisateur car l'urgence ressentie est telle que la seule logique de l'action nourrit, fédère et construit. Cette logique va reposer sur un savoir-faire et une méthode qui serviront de base aux initiatives politiques nécessaires.

Ainsi se crée un espace constitutif d'un État qui entretient une relation avec la société par d'autres voies que les mécanismes de la formulation politique. Voilà ce que pourrait recouvrir la réforme sociale: une possibilité de «cogestion» entre acteurs publics et acteurs privés, au
53. F. Ewald, «La politique sociale des opportunistes. 1879-1885», in S. Berstein et O. Rudelle, Le Modèle républicain, Paris, PUF, p. 187.

54. Dans l'esprit de la réflexion sur le processus de médiation, voir B. Jobert, Modes de médiations sociales et politiques publiques, L'Année sociologique, 40, 1990, pp. 155-178.

55. Voir E. Pisier-Kouchner, "Le service public entre libéralisme et collectivisme", Esprit, vol. $12, \mathrm{n}^{\circ} 2$, déc. 1983, pp. 9-19.

56. Voir R. Lenoir, «Groupes de pression et groupes consensuels », Actes de la recherche en sciences sociales, $\mathrm{n}^{\circ} 64,1986$, pp. $30-39$ 


\section{O S S I E R}

\section{Etatisations}

Martine Kaluszynski Réformer la société
57. P. Duran, «Pour une approche raisonnée des politiques publiques ", l'Année sociologique, 40, 1990, p. 247. point qu'il devient difficile de tracer les frontières de la sphère publique ${ }^{57}$. Ce qui serait en jeu ne serait donc pas tant l'expansion du public au détriment du privé, mais la question de la transformation des notions de privé et public. Au sein de cet espace associatif qui garde toute son autonomie et son regard critique par rapport aux politiques menées en matière de justice, il faudrait s'attacher précisément aux trois tendances ou trois milieux qui semblent très schématiquement se dessiner, en se neutralisant, dès les premières années de la SGP: les "philanthropes», les «professionnels» et les «politiques». Selon les périodes et le contexte, la résultante de ces forces en présence aboutira parfois à un équilibre ou une interaction, parfois à une prédominance ou à la disparition de l'une d'entre elles. Cette variation modulera et insufflera à la SGP son projet, son esprit et sa dynamique.

À tâtons, nous avons essayé de reconstituer les aspects qui permettent de caractériser l'espace du projet réformateur. De là nous pouvons procéder à une timide identification. Être un réformateur social ne relèverait pas d'une conviction politique, mais de l'adhésion à un projet mobilisateur organisé autour de préoccupations sociales, par le moyen de l'association et où le savoir-faire, le travail et l'organisation soudent et transcendent les clivages possibles. Une logique de l'action et du pragmatisme, la valorisation d'un savoir expert exercé en un espace privé «au détriment » d'un engagement public par son statut, sa forme et son espace. Un mode d'exercice du politique qui ne s'avoue pas sur ce registre, une forme apolitique de l'engagement qui a néanmoins pouvoir et capacité à bouleverser l'ordre social et politique. 